\title{
Mobile pediatric neurosurgery: rapid response neurosurgery for remote or urgent pediatric patients
}

\author{
Brian K. Owler, MBBS, FRACS, ${ }^{1,2}$ Kathryn A. Browning Carmo, BMED, FRACP, $, 3,3$ \\ Wendy Bladwell, BSc, MNurs, ${ }^{3}$ T. Arieta Fa'asalele, MBChB, ${ }^{3}$ Jane Roxburgh, BSc, MNurs, ${ }^{3}$ \\ Tina Kendrick, BNurs (Honours), MNurs, ${ }^{3}$ and Andrew Berry, MBBS, FRACP ${ }^{3}$
}

\begin{abstract}
1Discipline of Child Health and Paediatrics, Children's Hospital at Westmead Clinical School, University of Sydney; ${ }^{2}$ Department of Neurosurgery, ${ }^{4}$ Grace Centre for Newborn Intensive Care, Children's Hospital at Westmead, Sydney Children's Hospital Network, Sydney, Australia; ${ }^{3}$ Newborn and Paediatric Emergency Transport Service (NETS), New South Wales, Australia
\end{abstract}

OBJECT Time-critical neurosurgical conditions require urgent operative treatment to prevent death or neurological deficits. In New South Wales/Australian Capital Territory patients' distance from neurosurgical care is often great, presenting a challenge in achieving timely care for patients with acute neurosurgical conditions.

METHODS A protocol was developed to facilitate consultant neurosurgery locally. Children with acute, time-critical neurosurgical emergencies underwent operations in hospitals that do not normally offer neurosurgery. The authors describe the developed protocol, the outcome of its use, and the lessons learned in the 9 initial cases where the protocol has been used. Three cases are discussed in detail.

RESULTS Nine children were treated by a neurosurgeon at 5 rural hospitals, and 2 children were treated at a smaller metropolitan hospital. Road ambulance, fixed wing aircraft, and medical helicopters were used to transport the Newborn and Paediatric Emergency Transport Service (NETS) team, neurosurgeon, and patients.

In each case, the time to definitive neurosurgical intervention was significantly reduced. The median interval from triage at the initial hospital to surgical start time was 3:55 hours, (interquartile range [IQR] 03:29-05:20 hours). The median distance traveled to reach a patient was $232 \mathrm{~km}$ (range 23-637 km). The median interval from the initial NETS call requesting patient retrieval to surgical start time was 3:15 hours (IQR 00:47-03:37 hours). The estimated median "time saved" was approximately 3:00 hours (IQR 1:44-3:15 hours) compared with the travel time to retrieve the child to the tertiary center: 8:31 hours (IQR 6:56-10:08 hours).

CONCLUSIONS Remote urgent neurosurgical interventions can be performed safely and effectively. This practice is relevant to countries where distance limits urgent access for patients to tertiary pediatric care. This practice is lifesaving for some children with head injuries and other acute neurosurgical conditions.

http://thejns.org/doi/abs/10.3171/2015.2.PEDS14310

KEY WORDS neurosurgery; transfer; outcome; pediatric

$\mathrm{T}$ IME-CRITICal neurosurgical conditions such as traumatic or spontaneous intracranial hematoma (ICHs) require urgent operative treatment to prevent death or neurological deficits. $3,4,6,7,8$ Three children's hospitals in New South Wales (NSW) care for a population of 7.2 million people. ${ }^{1} \mathrm{NSW}$ is $800,628 \mathrm{~km}^{2}$ and surrounds the Australian Capital Territory (ACT), a $2366 \mathrm{~km}^{2}$ enclave of 376,500 people. ${ }^{1}$ Therefore, from the place of incident or injury, the distance to neurosurgical care is often great, presenting a challenge in achieving timely care for patients with acute neurosurgical conditions.

The Newborn and Paediatric Emergency Transport Service (NETS) is a dedicated intensive care retrieval service, using road and rotary and fixed wing vehicles (http:// www.nets.org.au). Over 254 hospitals in NSW/ACT call on NETS to assist children up to 16 years of age. The retrieval team normally comprises a specialist intensive care nurse and doctor. While this model works well for the majority

ABBREVIATIONS ACT = Australian Capital Territory; $\mathrm{AVM}=$ arteriovenous malformation; $\mathrm{EDH}=$ extradural hematoma; $\mathrm{EVD}=$ external ventricular drain; $\mathrm{GCS}=\mathrm{Glasgow}$ Coma Scale; ICH = intracranial hematoma; ICP = intracranial pressure; IQR = interquartile range; NETS = Newborn and Paediatric Emergency Transport Service; NSW = New South Wales; $\mathrm{PICU}=$ pediatric intensive care unit.

SUBMITTED July 9, 2014. ACCEPTED February 16, 2015.

INCLUDE WHEN CITING Published online June 19, 2015; DOI: 10.3171/2015.2.PEDS14310.

DISCLOSURE The authors report no conflict of interest concerning the materials or methods used in this study or the findings specified in this paper. 
of patients, for some patients, outcome has been compromised by the lack of timely access to specialized neurosurgical care. This has been due to the distance, delays in team or vehicle availability, inclement weather, and other logistical factors. Also, all fixed wing and many helicopter transfers require ground ambulance transport at one or both ends of the flight. Data reviewed from July 2007 to June 2009 identified 8 patients with time-critical head injuries, who required urgent surgery on admission to the tertiary center. Patients were located a median distance of $227 \mathrm{~km}$ (interquartile range [IQR] 61.0-469.3 km) from NETS. The median interval from incident to admission was 8:31 hours (IQR 6:56-10:08 hours) (http://www.nets. org.au).

The Neurosurgical Society of Australasia has made the following recommendations for management of a deteriorating patient with suspected $\mathrm{ICH}:{ }^{5} 1$ ) Transfer patient to a neurosurgeon within 2 hours, stabilize the airway, and administer intravenous $20 \%$ mannitol solution $(1 \mathrm{~g} / \mathrm{kg}$ body weight). Hypertonic saline is an alternative. 2) If patient transfer will take longer than 2 hours, bur hole exploration should be performed by the country practitioner or general surgeon on site in a regional hospital, where neurosurgery is not available. In some situations, it may be possible to arrange for a neurosurgeon to travel with a retrieval team to complete the operation.

The aformentioned protocol is usually carried out in the event of adult extraaxial collections. General surgeons in rural Australia have become increasingly uncomfortable with operating on children, and none of the patients in the following cases underwent bur hole procedures prior to the arrival of a neurosurgeon. The motivation for offering mobile pediatric neurosurgery was that previous experience indicated that bur holes performed by surgeons unaccustomed to pediatric neurosurgery were often inadequate to evacuate the hematoma and or created bleeding that was difficult to control.

Consequently, in July 2009, a protocol was developed to facilitate consultant neurosurgery locally. Children with acute, time-critical neurosurgical emergencies have undergone surgery in hospitals that do not offer neurosurgery. We describe the protocol that has been developed, the outcome of its use, and the lessons learned in the 9 initial cases in which the protocol has been used. Three cases are discussed in detail.

\section{Methods \\ Protocol Development}

There were several preconditions and logistical discussions that were required to enable the transport of a neurosurgeon to a patient to perform acute neurosurgery (Fig. 1). Firstly, clinical evidence, and preferably radiological evidence of the urgency and significant effectiveness of intervention, were required. In assessing a patient, there is a degree of subjectivity to the interpretation of the CT scans and the clinical urgency as determined by the neurosurgeon and the retrieval consultant on the initial call. A neurosurgeon needed to be available and free of on-call responsibilities or able to arrange cover of those responsibilities. A request was required for local hospital operating room staff, including an anesthetist and operating room nurses, to participate in surgery outside the scope of their normal hospital practice. These discussions were generally conducted by the neurosurgeon and the NETS retrieval consultant prior to or during the task preparation process for travel to the local hospital. In no case was an anesthetist required to travel to a remote hospital; local anesthetic consultants, with the support of the NETS team, were able to provide this service.

Figure 1 illustrates the decision-making process and all of the factors that must be considered by the NETS retrieval consultant and the neurosurgeons when utilizing the mobile pediatric neurosurgery protocol (http:// www.nets.org.au). Approval for network personnel to be released from hospital duties for retrieval service requires a phone call by the NETS consultant to notify the senior administrator of the Sydney Children's Hospital Network.

\section{Development of an Emergency Neurosurgical Retrieval Kit}

Care outside of the tertiary setting should be of a similar standard to that provided in a tertiary hospital; however, from the first case, significant deficiencies with the equipment at local hospitals were recognized. In a previous era, equipment was available for a local doctor to perform a bur hole procedure, but this equipment is no longer available. This led to the development of a specifically designed neurosurgical emergency kit by a representative of Medtronic Midas Rex in consultation with NETS and the pediatric neurosurgical team, an initiative funded by the Sydney Baseball Lions Club. The design specifications included readiness for immediate use, compliance with aircraft weight and size restrictions, utility in any standard operating theater, and wheeled mobility for optimal manual handling (Fig. 2). The kit, which is stored at the NETS base of operations, contains the equipment required to perform the bur hole procedure (Fig. 3), and it is authorized for use by designated and approved pediatric neurosurgeons in collaboration with NETS. Ethics approval for these case studies was obtained from the Sydney Children's Hospital Human Research Ethics Committee, and informed consent was not required.

\section{Results}

Nine children have been treated by a neurosurgeon at 5 rural hospitals, and 2 children were treated at smaller metropolitan hospitals. Road ambulance, fixed wing aircraft, and medical helicopters were used to transport the NETS team, neurosurgeon, and patient, in combination or singly.

In each case, the time to definitive neurosurgical intervention was significantly reduced by mobile neurosurgery, and in the 3 cases described below, the mobile protocol was considered lifesaving. The median interval from triage at the initial hospital to surgical start time was 3:55 hours, (IQR 3:29-5:20). The median distance traveled to reach a patient was $312 \mathrm{~km}$ (range $23-637 \mathrm{~km}$ ). Two patients (cases 4 and 8 ) required a fixed wing and a road trip initially to their regional base hospitals, $80 \mathrm{~km}$ away, to facilitate CT scanning and neurosurgery. Of note, the median interval from the initial NETS call requesting retrieval to surgical start time was under 4 hours, at 3:15 hours (IQR 00:47-3:37 hours). This was despite significant delays due to weather, long distances, aircraft delays, 


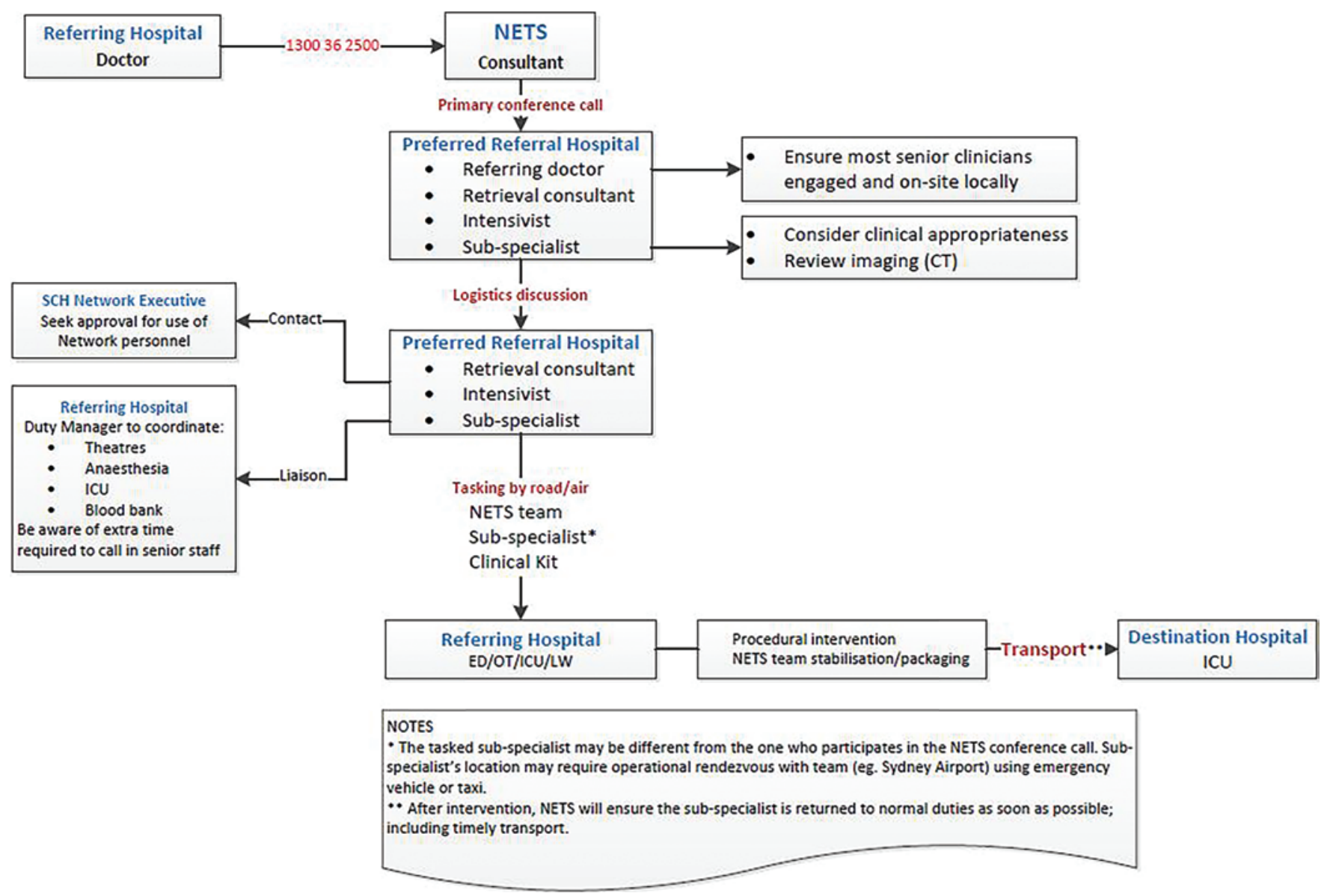

FIG. 1. Decision tree for utilizing mobile pediatric neurosurgery protocol. ED = emergency department; ICU = intensive care unit; $\mathrm{LW}=$ labor ward; OT = operating theater; SCH = Sydney Children's Hospital. Figure is available in color online only.

and interhospital transfer before surgery. The estimated median "time saved" was approximately 3:00 hours, (IQR 1:44-3:15 hours) as seen in Table 1.

In each case, surgery was performed without significant difficulty. In no case was the surgery considered to have been suboptimal or of lesser quality than that which would have been performed in a tertiary setting. The postoperative CT scans after transport to the tertiary center confirmed that the hematomas had been evacuated in each case.

\section{Illustrative Cases}

\section{Case 1}

A 2-year-old boy fell out of bed the evening before presentation. The following morning he was unresponsive, with irregular breathing and right-sided weakness. He was taken by ambulance to a regional base hospital (449 km from Sydney); his Glasgow Coma Scale (GCS) score was 6 . The right pupil was fixed and dilated, and the left pupil was poorly reactive. A large scalp hematoma was evident. He was sedated, ventilated, and given hypertonic saline. Cerebral CT demonstrated a large, right-sided extradural hematoma $(\mathrm{EDH})$, with a greater than 2-cm midline shift, complicated by a posterior cerebral artery territory infarction.
NETS was contacted at 5:01 AM while the ambulance was en route with the patient to the regional base hospital. An NETS team accompanied by a neurosurgeon was dispatched at 7:00 AM by fixed wing aircraft, arriving at the hospital at 8:39 AM.

A right temporal craniotomy was performed 3:35 hours after initial presentation. The intraoperative findings were a right temporal EDH with a single middle meningeal artery bleeding point identified and coagulated. Following the craniotomy, the patient's condition stabilized, and he was transported to a tertiary pediatric intensive care unit (PICU), arriving at 2:37 PM. A postoperative CT scan demonstrated complete evacuation of the $\mathrm{EDH}$, with a right posterior cerebral artery territory infarct consistent with previously raised intracranial pressure (ICP). Postoperative MRI revealed diffuse hyperintense areas in the right temporal and occipital regions, in the superior cerebellar regions on the left and posterior aspect of the midbrain, and also a small interhemispheric subdural hematoma on the left side.

The patient had a prolonged hospital stay requiring rehabilitation. Neurological deficits included a right hemiplegia, right cranial nerve III and VII palsies, and left homonymous hemianopia. 


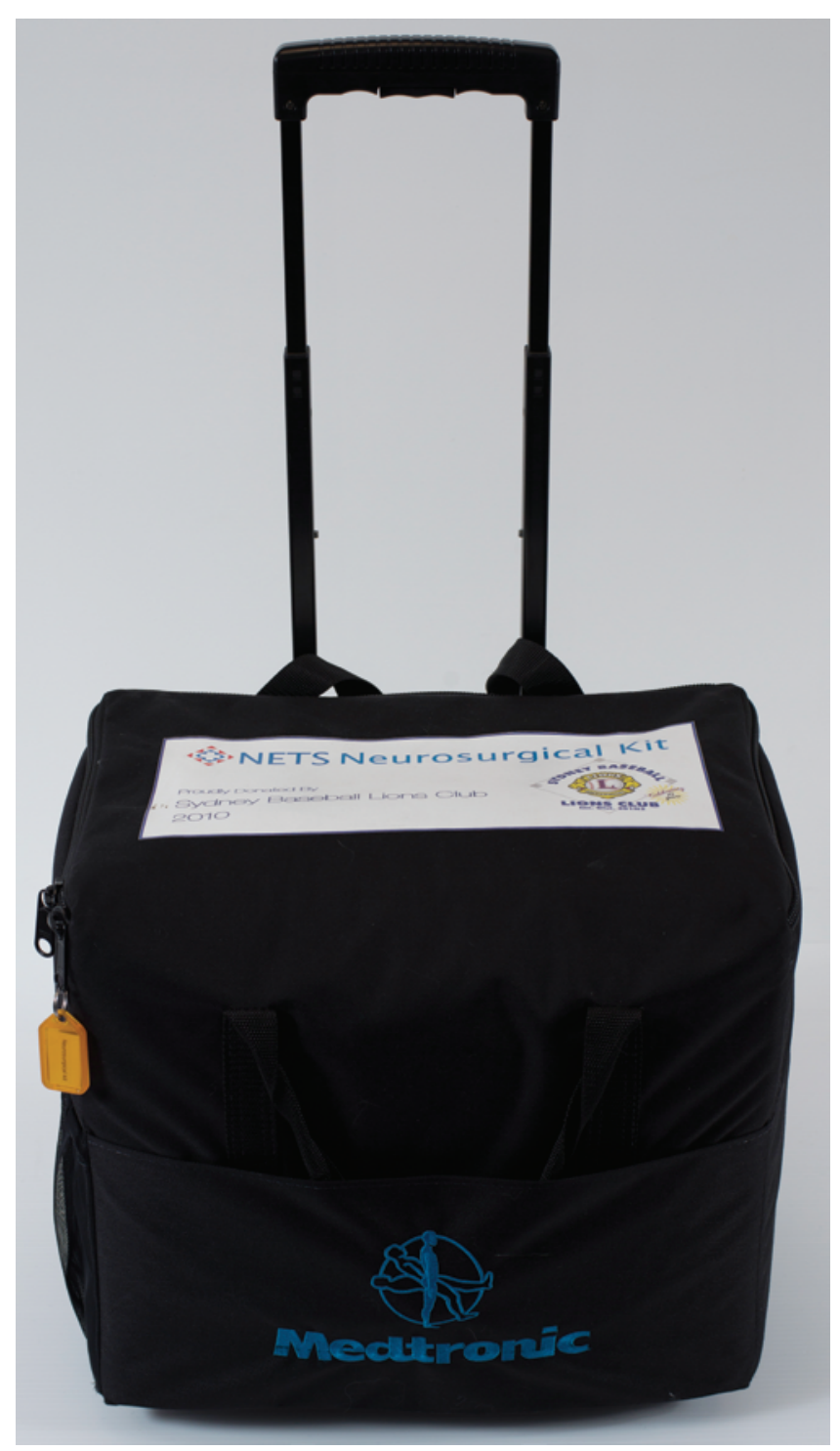

FIG. 2. Mobile pediatric neurosurgical kit. Figure is available in color online only.

\section{Case 2}

An 11-year-old girl had complained of headache the preceding night. The following morning she was having seizures and was taken to the emergency department at a large regional base hospital (199 km from Sydney). She presented at 11:50 AM with a dilated right pupil and was sedated, ventilated, and given mannitol. A cerebral CT scan demonstrated a large right ICH with midline shift and intraventricular extension (Fig. 4).

NETS was contacted at 12:41 PM.

Severe storms prevented the use of fixed or rotary wing aircraft; thus the neurosurgeon and retrieval team departed in the NETS road ambulance at 1:05 PM. and traveled under emergency driving conditions, arriving at the hospital at 3:46 PM.

While the team was en route, arrangements were made for the patient to be prepared for craniotomy. A right parietal craniotomy was performed. On opening the dura, the

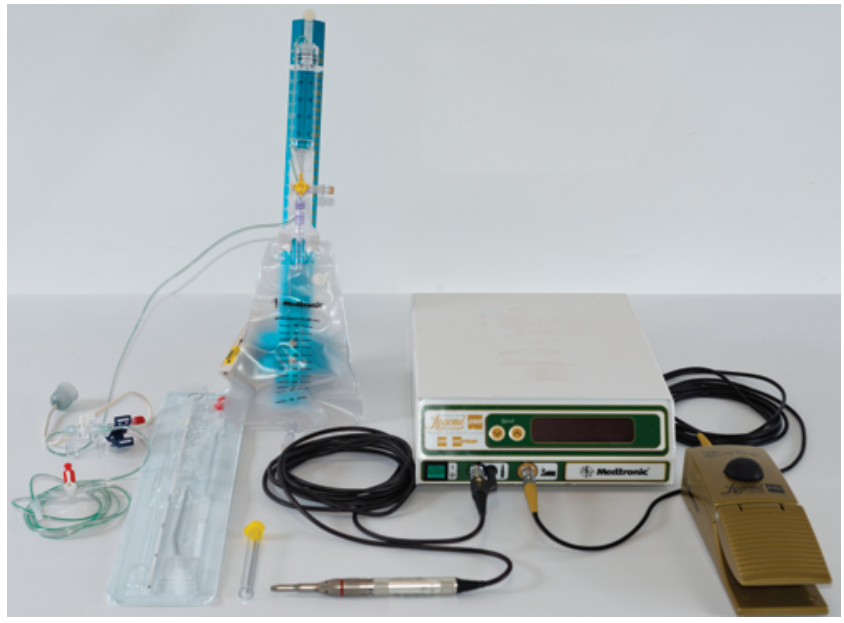

FIG. 3. Contents of the mobile neurosurgical kit. Figure is available in color online only.

thin cortical region directly over the hematoma was bulging under significant pressure. A large arterial draining vein was noted adjacent to the hematoma and was preserved. The hematoma was evacuated and the brain was decompressed by approximately 4:15 PM. Small bleeding vessels were coagulated and hemostasis was easily achieved. An external ventricular drain (EVD) was inserted and the bone flap was replaced using a small plating system.

At the end of the procedure, the patient was stable and was transported to the PICU by fixed wing air ambulance, arriving at the PICU at 10:05 PM.

Postoperative CT confirmed evacuation of the hematoma. The patient remained ventilated for 4 days. A digital subtraction angiogram confirmed the presence of a small right parietal arteriovenous malformation (AVM) (Spetzler-Martin Grade I). Following extubation she was alert with a GCS of 15 and with a dense left hemiplegia. An elective craniotomy and excision of the AVM was performed and confirmed by angiography. Over subsequent weeks there was marked improvement in her neurological deficit.

The time to decompression from the time of first call to NETS was 3:34 hours. Had the neurosurgeon not treated the child at the regional base hospital, the estimated time of surgery at the tertiary pediatric hospital would have been 8-9 hours. An additional 5 hours with significantly raised ICP and herniation would likely have resulted in poor neurological outcome or death.

\section{Case 3}

A 5-year-old boy developed a headache. He then collapsed and was taken to a large urban hospital, $23 \mathrm{~km}$ from NETS, arriving at 10:16 AM. His GCS score was 11, with pupils equal and reacting. A cerebral CT scan demonstrated a large right-sided $5 \times 3-\mathrm{cm}$ ICH in the temporoparietal area, extending into the occipital lobe, with significant mass effect and uncal herniation. During the $\mathrm{CT}$ scanning procedure there was rapid deterioration of the GCS score to 5. NETS were contacted at 11:07 AM. An NETS team and neurosurgeon, equipped with the neu- 
TABLE 1. Summary of logistical data for trips to and from the hospital where mobile pediatric neurosurgery was performed

\begin{tabular}{|c|c|c|c|c|c|c|}
\hline Case No. & Distance $^{*}(\mathrm{~km})$ & Transport Mode & Triage to MPNS (hrs) & $\begin{array}{c}\text { Initial NETS Call to MPNS } \\
\text { (hrs) }\end{array}$ & $\begin{array}{c}\text { Estimated Time to Op w/o } \\
\text { MPNS (hrs) } \dagger\end{array}$ & $\begin{array}{c}\text { Estimated "Time Saved" } \\
\text { (hrs) } \ddagger\end{array}$ \\
\hline 1 & 449 & Plane, road & $03: 29$ & 03:15 & $07: 22$ & 04:07 \\
\hline 2 & 232 & Plane, road & $03: 56$ & 03:05 & $06: 10$ & 03:05 \\
\hline 3 & 23 & Road & 01:17 & $00: 26$ & $01: 48$ & $01: 22$ \\
\hline 4 & 232 & Plane, road & $03: 40$ & $03: 33$ & 06:16 & $02: 43$ \\
\hline 5 & 23 & Road & $05: 20$ & $00: 47$ & $02: 22$ & $01: 35$ \\
\hline 6 & 384 & Plane, road & 03:55 & 03:37 & $06: 52$ & 03:15 \\
\hline 7 & 520 & Helicopter & $06: 36$ & $05: 37$ & 08:37 & 03:00 \\
\hline 8 & 637 & Plane, road & $05: 56$ & $05: 36$ & $09: 21$ & $03: 45$ \\
\hline 9 & 36 & Road & $03: 13$ & $00: 46$ & $02: 30$ & $01: 44$ \\
\hline Median & 232 & & $03: 55$ & 03:15 & $06: 16$ & 03:00 \\
\hline IQR & $(36.0-449.0)$ & & $(03: 29-05: 20)$ & $(00: 47-03: 37)$ & $(02: 30-07: 22)$ & $(01: 44-03: 15)$ \\
\hline
\end{tabular}

MPNS = mobile pediatric neurosurgery.

* Distance from tertiary hospital.

$\dagger$ Time from the initial NETS call to team arrival plus 1-hour retrieval of patient to return transport vehicle plus inbound travel time to tertiary center.

$\ddagger$ Difference between time from initial NETS call to tertiary center w/o MPNS and time from initial NETS call to arrival of MPNS.

rosurgical kit, were dispatched by road ambulance. The neurosurgeon reviewed the $\mathrm{CT}$ scan en route to the patient and recommended urgent evacuation of the ICH.

During the initial call, the pediatric intensivist advised that the patient be ventilated, given dexamethasone and mannitol, and be prepared for craniotomy. The elapsed time between the call to NETS to the start of surgery was 1:12 hours.

A small craniotomy was performed, and a large hematoma was evacuated. Large blood vessels in the depths of the hematoma were noted to appear abnormal and were directly coagulated, achieving hemostasis. An EVD was inserted.

At the end of the procedure, the patient was transported with NETS by road ambulance to a tertiary PICU. During transport, the patient exhibited a short period of hypertension, requiring EVD release with good effect.

The patient subsequently underwent digital subtraction angiography, and no vessel abnormalities were identified. The patient made a full neurological recovery and has not required further treatment.

\section{Discussion}

A number of basic principles guided development of this project. Firstly, the project is aimed at improving equity of access for patients to pediatric neurosurgical services. Secondly, the neurosurgical services provided should be of a tertiary level, with no compromise in quality of the care provided to patients. The use of a neurosurgeon in this activity should not compromise coverage of the tertiary hospital at which the neurosurgeon is based. Finally, the neurosurgical staff must be willing to travel and operate in conditions without their usual team.

There have been no major issues with equipment or staffing in the remote locations. One minor problem was the availability of suitable plating systems for replacement of the bone flap, which was resolved by adding a small plating system to the kit.
There were other logistical details that were addressed prior to establishing this service. Medico-legal indemnity for the neurosurgeon needed to be confirmed with the insurance providers.

Hospital accreditation was addressed through the wellestablished precedents as being an extension of an NETS team, appropriately tasked by the usual NETS process. Nonsurgical services provided by NETS are already pro-

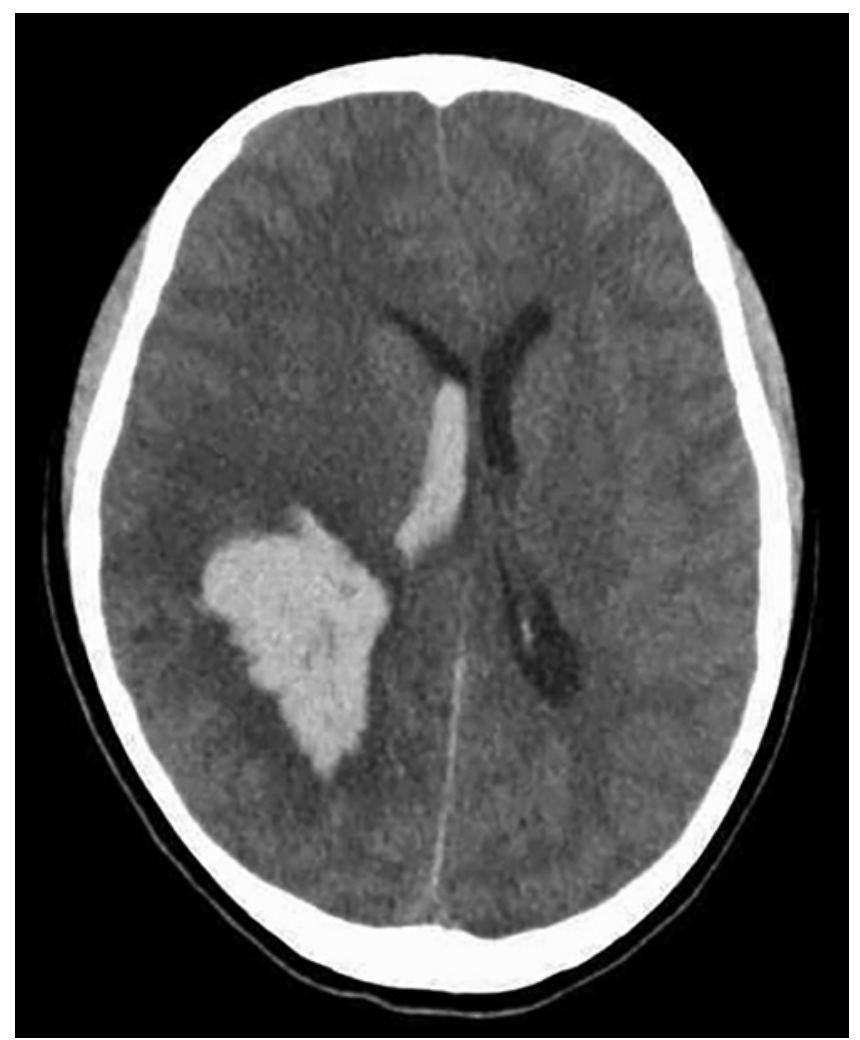

FIG. 4. Case 2. A CT image of an 11-year-old girl demonstrating right $\mathrm{ICH}$ with midline shift and intraventricular extension. 
vided by NETS doctors who have statewide accreditation. It is planned for surgery of this type to be included in service level agreements between referring hospitals and the NETS. Orientation to the aircraft and safety briefings for the neurosurgeons were also conducted.

The current protocol has been successful to date, with no patient complications or compromise in those cases where the protocol was used. Rather, we believe that in several cases the neurosurgical procedures were lifesaving. We have also demonstrated that mobile neurosurgery has decreased time to critical surgery from a median triage to tertiary admission from 06:16 hours (IQR 2:30-07:22 hours) down to 03:55 hours (IQR 03:29-05:20 hours).

Although there is no way in this case series to objectively determine what the outcomes would have been if the patients' surgeries had been delayed by retrieval, we believe they would have been significantly different. In a 2002-2004 retrospective review of data from Queensland, Australia, retrieved patients who required neurosurgery were delayed by a median of 04:46 hours and were more likely to die of their injury than those presenting directly to a hospital where neurosurgical services were available. In contrast, all 9 patients who received emergency bur holes in regional and rural hospitals by nonneurosurgeons survived, 8 with good outcomes. ${ }^{2}$

There are obvious questions about the role and viability of this model in the adult population. Given the increased incidence of urgent conditions in adults, there would be extensive issues with staffing and availability of personnel to provide such a service. ${ }^{1}$ The South Australian retrieval service documented 16 cases of adult mobile neurosurgery, which enabled better patient outcomes, where the patient was greater than 2 hours from the tertiary hospital. ${ }^{3}$ General surgeons are also more likely to be able and willing to perform decompressive surgery in adult patients. ${ }^{8}$ It has been the NETS's experience that for the pediatric population, general surgeons are increasingly less likely to feel comfortable intervening in this manner (http://www. nets.org.au).

This program may serve as a model of care for other conditions where the provision of care is time critical. Examples include acute ENT airway management as well as obstetric services for complex deliveries in remote locations.

The program will continue to be monitored to ensure that patient safety is not compromised and that outcomes are enhanced. Although we do not anticipate that the service will be required on a frequent basis, we believe the availability based on established protocols and essential equipment has not only saved lives and improved outcomes but will continue to do so in the future.

\section{Conclusions}

We have demonstrated that remote urgent neurosurgical interventions can be performed safely and effectively. This practice is particularly relevant to countries such as Australia, where distance limits urgent access for patients from peripheral hospitals to tertiary pediatric care. This practice is potentially lifesaving for some children with head injuries and other acute neurosurgical conditions.

\section{Acknowledgments}

We would like to acknowledge Dr. Robert Halliday for photography and Dr. Karen Walker for assistance with manuscript preparation.

\section{References}

1. Australian Bureau of Statistics: 3101.0 - Australian Demographic Statistics, Dec 2012. (http://www.abs.gov.au/AUS STATS/abs@.nsf/Lookup/3101.0Main+Features1Dec\%20 2012?OpenDocument) [Accessed April 17, 2015]

2. Deverill J, Aitken LM: Treatment of extradural haemorrhage in Queensland: interhospital transfer, preoperative delay and clinical outcome. Emerg Med Australas 19:325-332, 2007

3. Gilligan JE, Griggs WM, Jelly MT, Morris DG, Haslam RR, Matthews NT, et al: Mobile intensive care services in rural South Australia. Med J Aust 171:617-620, 1999

4. Lind CRP, Heppner PA, Robins TM, Mee EW: Transfer of intubated patients with traumatic brain injury to Auckland City Hospital. ANZ J Surg 75:858-862, 2005

5. Neurosurgical Society of Australia Inc: The Management of Acute Neurotrauma in Rural and Remote Locations. A Set of Guidelines for the Care of Head and Spinal Injuries. East Melbourne: Neurological Society of Australia Inc, 2009

6. Simpson DAHJ, Heyworth JS, McLean AJ, Gilligan JE, North JB: Extradural haemorrhage: strategies for management in remote places. Injury 19:307-312, 1988

7. Tasker RC, Morris KP, Forsyth RJ, Hawley CA, Parslow RC: Severe head injury in children: emergency access to neurosurgery in the United Kingdom. Emerg Med J 23:519-522, 2006

8. Treacy PJ, Reilly P, Brophy B: Emergency neurosurgery by general surgeons at a remote major hospital. ANZ J Surg 75:852-857, 2005

\section{Author Contributions}

Conception and design: Browning Carmo, Owler, Berry. Acquisition of data: Browning Carmo, Owler, Bladwell, Fa'asalele, Berry. Analysis and interpretation of data: Browning Carmo, Owler, Fa' asalele. Drafting the article: Browning Carmo, Owler, Fa'asalele, Roxburgh. Critically revising the article: Browning Carmo, Owler, Bladwell, Roxburgh, Kendrick, Berry. Reviewed submitted version of manuscript: all authors. Approved the final version of the manuscript on behalf of all authors: Browning Carmo. Statistical analysis: Fa' asalele. Administrative/ technical/material support: Browning Carmo, Bladwell,

Fa' asalele, Roxburgh. Main surgeon involved in the mobile neurosurgery service: Owler. Nursing care of patients on retrieval facilitating neurosurgery: Bladwell. Statewide director/facilitated development of protocol: Berry.

\section{Correspondence}

Kathryn Browning Carmo, Sydney Children's Hospital Network, P.O. Box 205, Westmead NSW 2145, Australia. email: kath. carmo@nets.org.au. 